\title{
Conditional probabilities with Dirac observables and the problem of time in quantum gravity
}

\author{
Rodolfo Gambini, ${ }^{1}$ Rafael A. Porto, ${ }^{2}$ Jorge Pullin, ${ }^{3}$ and Sebastián Torterolo ${ }^{1}$ \\ ${ }^{1}$ Instituto de Física, Facultad de Ciencias, Iguá 4225, esq. Mataojo, Montevideo, Uruguay \\ ${ }^{2}$ Department of Physics, University of California, Santa Barbara, California 93106, USA \\ ${ }^{3}$ Department of Physics and Astronomy, Louisiana State University, Baton Rouge, Louisiana 70803-4001, USA
}

(Received 25 September 2008; published 13 February 2009)

\begin{abstract}
We combine the "evolving constants" approach to the construction of observables in canonical quantum gravity with the Page-Wootters formulation of quantum mechanics with a relational time for generally covariant systems. This overcomes the objections levied by Kuchař against the latter formalism. The construction is formulated entirely in terms of Dirac observables, avoiding in all cases the physical observation of quantities that do not belong in the physical Hilbert space. We work out explicitly the example of the parametrized particle, including the calculation of the propagator. The resulting theory also predicts a fundamental mechanism of decoherence.
\end{abstract}

DOI: 10.1103/PhysRevD.79.041501

PACS numbers: 04.60.Pp, 03.65.Ta

In generally covariant systems, like general relativity, when one works out the canonical formulation the Hamiltonian is a constraint, i.e. it vanishes identically. This implies that the parameter that usually plays the role of time in canonical formulations is not adequate to describe the dynamics of the system. This constitutes one of the aspects of the "problem of time" for generally covariant systems (see Kuchař [1] for a good review). Page and Wootters proposed an approach to deal with this issue [2]. The proposal consists in building a quantum theory of the system of interest by promoting all variables of the system to quantum operators and then choosing one of the variables to be a "clock" and computing conditional probabilities for the other variables to take certain values when the clock takes a given value. This proposal ran into technical difficulties when applied in detail to constrained systems, as emphasized by Kuchař [1]. Basically, the problem consists in what to choose as the variables to be promoted to operators, in particular, which one to choose as a clock. In a constrained system the physically observable variables are those that have vanishing Poisson brackets with the constraints (this implies they are invariant under the symmetries of the theory, they are "gauge invariant"). However, if one of the constraints is the Hamiltonian, then quantities that have vanishing Poisson brackets with it do not evolve and therefore are poor candidates for being clocks. Page and Wootters tried to circumvent this by considering "kinematical" variables (that do not have vanishing Poisson brackets with the constraints and therefore appear "to evolve"). But this brings about other problems. Such variables can be promoted to quantum operators acting on the space of wave functions that are not necessarily annihilated by the constraints ("kinematical Hilbert space"). Within such space, the states that are annihilated by the constraints are usually distributional (at least in simple examples that can be worked out explicitly). Therefore they may not admit a probabilistic interpretation. Kuchař showed, by analyzing the example of a parametrized particle, that these issues had as a consequence the construction of propagators that "do not propagate" and therefore the resulting quantum theory is not realistic.

Here we would like to revisit the Page-Wootters construction but using a different set of physical quantities. The quantities we will choose are relational Dirac observables such as the "evolving constants" introduced in [3] (an idea that goes back to DeWitt, Bergmann, and Einstein). The proposal can be summarized as follows. In a totally constrained theory, the values of fields are not physically observable. On the other hand, if one chooses a one-parameter family of observables such that their value coincides with the value of a dynamical variable when the parameter takes the value of another dynamical variable, which one uses to characterize the evolution, such observables can be used in the Page-Wootters construction. They have the advantage that there is a sense in which they "evolve." That is, unlike the proposal of Rovelli, we will not consider the "parameter" to be the physical time, but we will use it to make sense of the conditional probabilities that arise in the Page-Wootters formulation when one introduces a real quantum clock. In fact at the end of the day the parameter drops out from the formulation, and one integrates over all possible values of it (if one has more than one constraint, one needs to introduce more parameters). Therefore, one does not need to observe any dynamical variable that is not quantum mechanical or is not a Dirac observable. We will show in an example that this construction can be carried out in detail.

Let us start by defining the evolving constants in a classical theory. Following Rovelli we consider a totally constrained system with a phase space $q^{i}, p^{i}$. We now pick a parameter we call $t$. We are interested in defining a oneparameter family of Dirac observables that reproduces the value of one of the dynamical variables, for instance $q^{i}$, when another variable playing the role of a clock takes the value $t$. For concreteness, we can choose $q^{1}$ to play the role of a clock. We denote the one-parameter family of Dirac 
observables $Q^{i}(t)=Q^{i}\left(t, q^{n}, p^{n}\right)$. These have vanishing Poisson bracket with the Hamiltonian constraint, $\left\{Q^{i}\left(t, q^{n}, p^{n}\right), C\left(q^{n}, p^{n}\right)\right\}=0$. They are also such that if one evaluates $Q^{i}\left(t, t, q^{2} \ldots q^{n}, p^{n}\right) \equiv q^{i}$. (We refer to the observables as $Q^{i}$ for simplicity, they can include momenta as well, but they must have vanishing Poisson brackets with the clock variable, an assumption that may be relaxed with further elaboration).

We then proceed to quantize the variables of the problem. Namely, we promote all $Q^{i}(t)$ 's and $P^{i}(t)$ 's to quantum self-adjoint operators acting on a Hilbert space of wave function $\Psi\left(q^{i}\right)$ that are annihilated by the constraints. The variable $t$ will remain classical. In realistic situations, like general relativity, this is convenient since usual choices of "time" are given by global variables that are not easily associated with a quantum operator to begin with. The restriction to self-adjoint operators limits importantly the choices of possible parameters $t$, as was discussed in [4]. In particular, if one does not insist on self-adjointness one runs into problems in the definition of conditional probabilities (related to the "false tracks" discussed in [5]). If the quantization can be accomplished, then one can introduce a basis of eigenstates (parametrized by $t$ ) of the evolving constants and introduce projectors that materialize the physical properties associated with each of the evolving constants.

Having quantized the evolving constants, we will choose one of the variables $Q^{i}(t)$ to be a quantum clock, and we will call it $T(t)$. We then introduce the conditional probability as in $[6,7]$ :

$$
\begin{aligned}
P\left(Q^{i}\right. & \left.=Q_{0}^{i} \mid T=T_{0}\right) \\
& \equiv \lim _{\tau \rightarrow \infty} \frac{\int_{-\tau}^{\tau} d t \operatorname{Tr}\left(P_{Q_{0}^{i}}(t) P_{T_{0}}(t) \rho P_{T_{0}}(t)\right)}{\int_{-\tau}^{\tau} d t \operatorname{Tr}\left(P_{T_{0}}(t) \rho\right)},
\end{aligned}
$$

where $P_{Q_{0}^{i}}(t)$ is the projector on the eigenspace associated with the eigenvalue $Q_{0}^{i}$ at time $t$ and similarly for $P_{T_{0}}(t)$. These conditional probabilities are positive and add to one. At this stage $\rho$ is the density matrix of the total system. To make contact with usual expressions later on, we will assume a specific form of the density matrix.

By construction the conditional probability is a gauge invariant quantity since the density matrix, $\rho$ in the above expression, is assumed to be annihilated by the constraints, e.g. $\hat{C} \rho=0$. Note that we are treating the variable $t$ as an unobservable quantity and summing over all possible values of it. This picture is much more satisfactory than the one that emerges from considering evolving constants alone without the conditional probability interpretation, since in that picture one assumes that a quantum variable like $q^{1}$ takes a definite classical value. This would not usually be the case since $q^{1}$ has a nonvanishing Poisson bracket with the constraint, and on the constraint surface we expect $q^{1}$ to have infinite uncertainty. Returning to the above expression, it should be noted that the improper limits of integration may cause problems at the time of computing the conditional probabilities. This can be controlled by simply considering integrals in temporal domains that are much larger than the region of physical interest.

It is worthwhile expanding on the meaning of the probabilities (1) since there has been some confusion in the literature [8]. One may interpret that the numerator of (1) is the sum of joint probabilities of $O$ and $T$ for all values of $t$. This would be incorrect since the events in different $t$ 's are not mutually exclusive. The probability (1) corresponds to a physically measurable quantity, and that such quantity is actually the only thing one can expect to measure in systems where one does not have direct access to the "ideal" time $t$. The experimental setup we have in mind is to consider an ensemble of noninteracting systems with two quantum variables each to be measured, $O$ and $T$. Each system is equipped with a recording device that takes a single snapshot of $O$ and $T$ at a random unknown value of the ideal time $t$. One takes a large number of such systems, launches them all in the same quantum state, "waits for a long time," and concludes the experiment. The recordings taken by the devices are then collected and analyzed all together. One computes how many times $n\left(T_{j}, O_{j}\right)$ each reading with a given value $T=T_{j}, O=O_{j}$ occurs (to simplify things, for the moment let us assume $T, O$ have discrete spectra; for continuous spectra one would have to consider values in a small finite interval of the value of interest). If one takes each of those values $n\left(T_{j}, O_{j}\right)$ and divides them by the number of systems in the ensemble, one obtains, in the limit of infinite systems, a joint probability $P\left(O_{j}, T_{j}\right)$ that is represented by the above expression.

We can then write the conditional probabilities that yield the correlation functions (propagators), namely, the probability that the system was observed at $Q_{1}^{i}$ at time $T_{1}$ and it will be observed at $Q_{2}^{i}$ at time $T_{2}$ as

$$
\begin{aligned}
& P\left(Q_{2}^{i} \mid T_{2}, Q_{1}^{i}, T_{1}, \rho\right) \\
& \quad \equiv \lim _{\tau \rightarrow \infty} \frac{\int_{-\tau}^{\tau} d t \int_{-\tau}^{\tau} d t^{\prime} \operatorname{Tr}\left(P_{Q_{2}^{i}, T_{2}}(t) P_{Q_{1}^{i}, T_{1}}\left(t^{\prime}\right) \rho P_{Q_{1}^{i}, T_{1}}\left(t^{\prime}\right)\right)}{\int_{-\tau}^{\tau} d t \int_{-\tau}^{\tau} d t^{\prime} \operatorname{Tr}\left(P_{T_{2}}(t) P_{Q_{1}^{i}, T_{1}}\left(t^{\prime}\right) \rho P_{Q_{1}^{i}, T_{1}}\left(t^{\prime}\right)\right)} .
\end{aligned}
$$

This is the standard definition of a propagator associated with a history once a reduction postulate after the measurement of $Q_{1}^{i}, T_{1}$ (see [6]) is assumed. Notice that, in particular, no assumption about the relative ordering of the unobservable variables $t$ and $t^{\prime}$ is needed. We will show that it yields the correct propagator in an example.

Until now, recovering the correct propagator has been problematic in the conditional probability approach. For instance, Kuchař [1] computed a similar expression using the original Page and Wootters [2] prescription (in that case, however there is no $t$ or $t^{\prime}$ and no integrals over them) where the quantities $Q^{i}$ were kinematical operators that did not commute with the constraint and showed that one 
obtained an incorrect propagator. Essentially, the system did not move, the propagator being proportional to a Dirac delta function, e.g. $\delta\left(Q_{2}^{i}-Q_{1}^{i}\right) \delta\left(T_{2}-T_{1}\right)$. Page [9] has responded to this criticism by claiming that in the conditional probability framework one cannot compute two time probabilities. We believe that the framework can indeed accommodate such probabilities and therefore becomes more powerful when formulated in terms of evolving constants and indeed yields the correct propagators.

The example we will consider is a simple model of two noninteracting nonrelativistic free particles in one spatial dimension that has been "parametrized," that is, Newtonian time is introduced as a canonical variable conjugate to the energy. The reader may question how relevant these simplified examples are to the issue of interest, namely, the problem of time in quantum gravity. To quote Kuchař [1] "The nature of the conditional probability interpretation is so clear from these examples that it is hardly necessary to spell out how the formalism looks in quantum gravity". The reader will confirm this point of view while seeing how one gets the result for the propagator virtually without using any special features of the model in question. In particular, although the model does have a naturally defined time variable, we only use it to easily construct the evolving constants. The latter are known to exist in many examples (e.g. [4]) where there is no natural decomposition of the constraint into the " $p^{0}+H$ " form.

The system has three configuration variables $q_{0}, q_{1}, q_{2}$ and the corresponding canonical momenta $p_{0}, p_{1}, p_{2}$. There is a constraint $\phi=p_{0}+p_{1}^{2} /\left(2 m_{1}\right)+p_{2}^{2} /\left(2 m_{2}\right)$. The gauge invariant quantities, which have vanishing Poisson brackets with the constraint, are $Q_{1}=$ $q_{1}-p_{1} q_{0} / m_{1}$ and $Q_{2}=q_{2}-p_{2} q_{0} / m_{2}$ and $p_{1}$ and $p_{2}$. These Dirac observables represent the initial position and momenta of the particles. We then define evolving constants $X_{1}(t)=Q_{1}+p_{1} t / m_{1}$ and $X_{2}(t)=Q_{2}+p_{2} t / m_{2}$. We can check that they have a vanishing Poisson bracket with the constraint and that when $t=q_{0}$ then $X_{1}(t=$ $\left.q_{0}\right)=q_{1}$ and $X_{2}\left(t=q_{0}\right)=q_{2}$. The quantization of the model is immediate [4]. The states that are annihilated by the quantum version of the constraints are given by $\psi\left(p_{1}, p_{2}\right)$ times a prefactor $\delta\left(p_{0}+p_{1}^{2} /\left(2 m_{1}\right)+\right.$ $\left.p_{2}^{2} /\left(2 m_{2}\right)\right)$ and the Hilbert space is that of square integrable functions $\psi\left(p_{1}, p_{2}\right)$, or equivalently in Fourier space by functions $\tilde{\psi}\left(q_{1}, q_{2}\right)$. The resulting states are normalizable with respect to the Rieffel induced inner product. In this Hilbert space the evolving constants are well defined operators. Their common eigenstates are of the product form,

$$
\begin{aligned}
\psi_{x_{1}, x_{2} ; t} & =\left\langle p_{1}, p_{2} \mid x_{1}, x_{2} ; t\right\rangle \\
& =\frac{1}{2 \pi} \exp \left(-i\left[p_{1} x_{1}+p_{2} x_{2}-t\left(\frac{p_{1}^{2}}{2 m_{1}}+\frac{p_{2}^{2}}{2 m_{2}}\right)\right]\right),
\end{aligned}
$$

with eigenvalues $x_{1}, x_{2}$ for $\hat{X}_{1}, \hat{X}_{2}$, at some value of $t$. With these we can construct the projectors that appear in the conditional probability, $P_{x_{1}}(t)=\int_{x_{1-}}^{x_{1+}} d z_{1} \int_{-\infty}^{\infty} d z_{2} \times$ $\left|z_{1}, z_{2} ; t\right\rangle\left\langle z_{1}, z_{2} ; t\right|$ and similarly for $P_{x_{2}}(t)$. The limits of integration $x_{i \pm}$ correspond to $x_{i} \pm \Delta x_{i} / 2$ where $\Delta x_{i}$ is introduced since, if one is dealing with variables that have continuum spectrum, one cannot ask for "the probability that $q_{i}$ takes a given value $x_{i}$," but rather within an interval of width $\Delta x_{i}$ centered at such value. Let us consider a physical state given by a Gaussian for both variables centered at two distant phase-space points $x_{1}^{0}$ and $x_{2}^{0}$ and $p_{1}^{0}$ and $\quad p_{2}^{0}$, e.g. $\rho_{0}=\left|\psi_{0}\right\rangle\left\langle\psi_{0}\right|$, with $\tilde{\psi}_{0}\left(q_{1}, q_{2}\right)=$ $\prod_{j=1}^{2} \exp \left(-\left(q_{j}-x_{j}^{0}\right)^{2} / \Delta_{j}^{2}+i p_{j}^{0} q_{j}\right)$. We can then compute the quantity in the numerator of (1) (notice that the denominator is just given by the numerator integrated in $Q_{2}^{i}$ from $-\infty$ to $\infty$, so for brevity we only show explicit calculations for the numerator),

$$
\begin{aligned}
\operatorname{Num}( & \left.P\left(x_{2}^{\prime} \mid x_{1}^{\prime}, x_{2}, x_{1}\right), \rho_{0}\right) \\
= & \int_{0}^{\tau} d t^{\prime} d t \int_{x_{2-}}^{x_{2+}} d y_{2} d z_{2}\left\langle x_{2}^{\prime}, t^{\prime} \mid y_{2}, t\right\rangle \psi_{y_{2}, t}^{0}\left(\psi_{z_{2}, t}^{0}\right)^{*} \\
& \times\left\langle z_{2}, t \mid x_{2}^{\prime}, t^{\prime}\right\rangle \int_{x_{1-}}^{x_{1+}} d y_{1} d z_{1}\left\langle x_{1}^{\prime}, t^{\prime} \mid y_{1}, t\right\rangle \psi_{y_{1}, t}^{0},\left(\psi_{z_{1}, t}^{0}\right)^{*} \\
& \times\left\langle z_{1}, t \mid x_{1}^{\prime}, t^{\prime}\right\rangle,
\end{aligned}
$$

where $\psi_{w_{i}, t}^{0} \equiv\left\langle w_{i}, t \mid \psi_{0}^{i}\right\rangle$ for $i=1,2$, and we have used the fact that the density matrix for this model is of direct product form, namely $\left|\psi_{0}\right\rangle=\left|\psi_{0}^{1}, \psi_{0}^{2}\right\rangle$. Usually one would like to consider systems with this property which implies that the system under study and the clock do not interact (we are choosing $x_{1}$ as the clock variable). The interval $\Delta x_{1}$ must be taken much larger than $\Delta_{1}$ the width of the Gaussian in the state in order for the measurement of the clock variable not to "destroy the state of the clock." A measurement with more precision implies a faster loss of the (desired) classicality of the clock. In the case of $x_{2}$ we assume we are studying a microscopic variable $\left(m_{2} \ll\right.$ $m_{1}$ ), i.e. that is behaving quantum mechanically, therefore we may and will assume $\Delta x_{2}$ much smaller than the width of the Gaussian $\Delta_{2}$ to simplify the calculation of the integrals by substituting mean values. Carrying out the integrations explicitly,

$$
\begin{aligned}
\operatorname{Num} & \left.P\left(x_{2}^{\prime} \mid x_{1}^{\prime}, x_{2}, x_{1}\right), \rho_{0}\right) \\
\sim & \int_{0}^{\tau} d t^{\prime} d t\left|\left\langle x_{2}^{\prime}, t^{\prime} \mid x_{2}, t\right\rangle\right|^{2}\left|\psi_{x_{2}, t}^{0}\right|^{2} \Delta x_{2}^{2} \Theta_{\Delta x_{1}} \\
& \times\left(\bar{x}_{1}^{0}-\frac{p_{1}}{m_{1}} t-x_{1}\right)\left|\psi_{x_{1}^{\prime}, t^{\prime}}^{0}\right|^{2},
\end{aligned}
$$

where $\Theta$ is a rectangular function that is unity in the interval of width $\Delta x_{1}$ around its argument and zero otherwise. We have assumed that evolution times are small such that the value of $\Delta x_{1}$ does not change significantly. We have approximated the integrals in $y_{1}$ and $z_{1}$ by integrals 
from $-\infty$ to $\infty$ since the Gaussian has a smaller support than the region of integration. The $\Theta$ function arises since the approximation is good only if the peak of the Gaussian is within the integration region, otherwise the integral is close to zero. Putting together numerator and denominator we get

$$
\begin{aligned}
& P\left(x_{2}^{\prime} \mid x_{1}^{\prime}, x_{2}, x_{1}, \rho_{0}\right) \\
& \quad \sim \lim _{\tau \rightarrow \infty} \int_{0}^{\tau} d t^{\prime}\left|\left\langle x_{2}^{\prime}, t^{\prime} \mid x_{2}, t\left(x_{1}\right)\right\rangle\right|^{2} \mathcal{P}_{x_{1}^{\prime}}\left(t^{\prime}\right) \Delta x_{2},
\end{aligned}
$$

where $t\left(x_{1}\right)$ is the central value of $t$ determined by the $\Theta$ function and $\mathcal{P}_{x_{1}^{\prime}}\left(t^{\prime}\right) \equiv \operatorname{Tr}\left(P_{x_{1}^{\prime}}\left(t^{\prime}\right) \rho_{0}\right) / \int_{-\infty}^{\infty} d t \operatorname{Tr}\left(P_{x_{1}^{\prime}}(t) \rho_{0}\right)$ can be interpreted as the probability that the external (unobservable) time $q^{0}$ is $t^{\prime}$ when the variable we take as clock reads $x_{1}^{\prime}$. This would be controlled by the position of the peak and width of the Gaussian in the quantum state we chose. If instead of a Gaussian one had a Dirac delta, then we would recover the correct ordinary nonrelativistic propagator, $P\left(x_{2}^{\prime} \mid x_{1}^{\prime}, x_{2}, x_{1}, \rho_{0}\right) \sim\left\langle x_{2}^{\prime}, t^{\prime}\left(x_{1}^{\prime}\right) \mid x_{2}, t\left(x_{1}\right)\right\rangle \Delta_{2}$, where $t\left(x_{1}\right)$ is determined by our choice of initial state to approximate the ordinary nonrelativistic time corresponding to the position $x_{1}$. The resulting expression is an approximation to the integral in $x_{2}$ of the ordinary propagator, therefore the factor $\Delta x_{2}$. As is expected in relational treatments, one only obtains the traditional propagator at leading order. The use of real clocks leads to loss of quantum coherence, as is well known [7], and therefore to corrections to the ordinary propagator. The origin of the loss of coherence is that, although the evolution is unitary in terms of the parameter $t$, it is not in terms of the real clock $T$. Even if one starts with a pure state initially, the lack of a perfect correlation of the variables $T$ and $t$ will lead to a statistical mixture. Notice that up to now discussions of loss of coherence due to real clocks did not involve the presence of constraints, since they were framed for the gravitational case in the context of uniform and consistent discretizations [6], where constraints are eliminated. Here we confirm not only the presence but the inevitability of these effects in totally constrained systems like general relativity.

Let us sketch how the above proposal could be implemented in the case of general relativity. We consider the theory in vacuum coupled to a clock. We characterize the clock by its worldline $X^{\mu}(\tau)$ and $T(\tau)$ its proper time. The action is the Einstein-Hilbert action for general relativity plus a term for the clock of the form $S=$ $-m \int d \tau \sqrt{-\dot{X}^{\mu} \dot{X}^{\nu} g_{\mu \nu}(X(\tau))-\dot{T}^{2}}$ where the dots mean total derivative with respect to the parameter $\tau$, and $m$ is the mass of the clock. The equations of motion state that
$X^{\mu}$ is a geodesic of the metric $g_{\mu \nu}$ and an equation stating that $T$ is proportional to the proper time. As usual, we are assuming that the clock is a probe and therefore ignore backreaction. Classically this is certainly a good approximation. In this system we have only introduced a clock, not a complete coordinate system; one can ask only certain relational questions. For instance, what is the value of a geometric scalar (e.g. the Kretschmann invariant) $K$ at the space-time position of the clock when the clock measures a given value of time $T$. To complete our proposal, one needs to find evolving constants $S$, functions of the metric, and its first derivatives, parametrized with four real parameters $x^{\mu}$ such that when they equal certain combinations of the metric and its derivatives the evolving constants reproduce the geometric quantity $S$ we want to measure. The explicit construction of these quantities in general relativity can be onerous, but progress can be done by perturbative techniques, for example (see [10]). One can then define the relational probabilities that the geometric quantity of interest takes the value $S_{0}$ when the clock measures time $T_{0}$,

$$
P\left(S_{0} \mid T_{0}\right)=\frac{\int d^{4} x \operatorname{Tr}(\sqrt{-g} \rho) \operatorname{Tr}\left(P_{S_{0}}(x) P_{T_{0}}(x) \rho P_{T_{0}}(x)\right)}{\int d^{4} x \operatorname{Tr}(\sqrt{-g} \rho) \operatorname{Tr}\left(P_{T_{0}}(x) \rho\right)} .
$$

Defining a propagator needs more work, namely, setting up a full coordinate system (i.e. introducing rulers in addition to clocks or considering a cloud of clocks as in [11]). The calculational complexity would be important but modern loop quantum gravity techniques may allow a proper calculation (the Marseille group has developed an attractive framework for the discussion of propagators in loop quantum gravity [12]). The expressions of the conditional probabilities in a situation like general relativity will not only include loss of coherence in time but also spatially, as has been analyzed in field theory in [13].

Summarizing, we have shown that one can formulate a completely relational picture of evolution in generally covariant systems framed entirely in the physical space of states and that yields the correct propagators in model systems and opens the possibility of assigning probabilities to histories and consistently characterizing the dynamics of quantum general relativity. The resulting theory also predicts a fundamental mechanism of decoherence similarly as the one originally discussed in [6].

We wish to thank Don Marolf for detailed comments. This work was supported in part by Grant No. NSF-PHY0650715, funds of the Hearne Institute for Theoretical Physics, FQXi, RPFI-06-18, CCT-LSU, the University of California, Pedeciba, and PDT (Proyecto 63/076) (Uruguay). 


\section{CONDITIONAL PROBABILITIES WITH DIRAC ...}

[1] K. Kuchař, in Proceedings of the 4th Canadian Conference on General Relativity and Relativistic Astrophysics, edited by G. Kunstatter, D. Vincent, and J. Williams (World Scientific, Singapore, 1992).

[2] D. N. Page and W. K. Wootters, Phys. Rev. D 27, 2885 (1983).

[3] C. Rovelli, Phys. Rev. D 43, 442 (1991).

[4] R. Gambini and R. A. Porto, Phys. Rev. D 63, 105014 (2001).

[5] F. Hellmann, M. Mondragon, A. Perez, and C. Rovelli, Phys. Rev. D 75, 084033 (2007).

[6] R. Gambini, R. Porto, and J. Pullin, New J. Phys. 6, 45 (2004).
PHYSICAL REVIEW D 79, 041501(R) (2009)

[7] R. Gambini, R. Porto, and J. Pullin, Gen. Relativ. Gravit. 39, 1143 (2007).

[8] C. Anastopoulos and B. L. Hu, J. Phys. Conf. Ser. 67, 012012 (2007).

[9] D. N. Page, in Physical Origins of Time Asymmetry, edited by J.J. Halliwell et al. (Cambridge University Press, Cambridge, England, 1993).

[10] B. Dittrich, Classical Quantum Gravity 23, 6155 (2006).

[11] C. Rovelli, Classical Quantum Gravity 8, 297 (1991).

[12] J. Engle, R. Pereira, and C. Rovelli, Phys. Rev. Lett. 99, 161301 (2007).

[13] R. Gambini, R. A. Porto, and J. Pullin, Int. J. Mod. Phys. D 15, 2181 (2006); Phys. Lett. A 372, 1213 (2008). 